\title{
Fixed point results via altering distance functions in relational fuzzy metric spaces with application
}

\author{
Ayush Bartwal*, R.C. Dimri, Shivam Rawat
}

\begin{abstract}
Some fixed point theorems are developed in fuzzy metric spaces using an altering distance function under binary relationship. We ensure the existence and uniqueness of the solution to ordinary differential equation using our results. We also give a non-trivial example to illustrate our primary result. Our results strengthen and extend the Theorem 3.1 of Shen et al. (Applied Mathematics Letters, 25 (2012), 138-141).
\end{abstract}

\section{INTRODUCTION AND PRELIMINARIES}

In 1960, Schweizer and Sklar [22] introduced the notion of continuous triangular norm as:

Definition 1 ([22]). A binary operation $*:[0,1] \times[0,1] \rightarrow[0,1]$ is said to be a continuous triangular norm (t-norm) if $*$ satisfies the following conditions:

(i) $\rho * \sigma=\sigma * \rho$ and $\rho *(\sigma * \varsigma)=(\rho * \sigma) * \varsigma$, for all $\rho, \sigma, \varsigma \in[0,1]$;

(ii) $*$ is continuous;

(iii) $1 * \rho=\rho$, for all $\rho \in[0,1]$;

(iv) $\rho * \sigma \leq \varsigma * \tau$, whenever $\rho \leq \varsigma$ and $\sigma \leq \tau$, for all $\rho, \sigma, \varsigma, \tau \in[0,1]$.

Example 1. $\rho * \sigma=\rho \sigma$ and $\rho * \sigma=\min \{\rho, \sigma\}$ are two basic examples of continuous t-norm, named as product t-norm and minimum t-norm respectively.

If $u_{1}, u_{2}, \ldots, u_{n} \in[0,1]$, then $*_{i=1}^{n} u_{i}=u_{1} * u_{2} * \cdots * u_{n}$. For every $u \in[0,1]$, the sequence $\left\{*_{i=1}^{n} u_{i}\right\}_{n=1}^{\infty}$ is defined by $*^{1} u=u$ and $*^{n+1} u=\left(*^{n} u\right) * u$, for all $n \geq 1$. If the sequence $\left\{*_{i=1}^{n} u_{i}\right\}_{n=1}^{\infty}$ is equicontinuous at $u=1$ then the t-norm $*$ is said to be of H-type (see [10]).

2020 Mathematics Subject Classification. Primary: 47H10; Secondary: 54H25.

Key words and phrases. Fuzzy metric spaces, fixed point, binary relation.

Full paper. Received 2 June 2021, accepted 2 September 2021, available online 1 October 2021.

*Corresponding Author 
Proposition 1 ([9]). Let $\left\{\alpha_{n}\right\}_{n \in \mathbb{N}} \in[0,1]$ be a sequence which converges to 1 and $t$-norm $*$ is of H-type. Then

$$
\lim _{n \rightarrow \infty} *_{i=n}^{\infty} \alpha_{i}=*_{i=1}^{\infty} \alpha_{i+1}=1 .
$$

In 1975, Kramosil and Michalek [14] introduced the notion of fuzzy metric spaces using the theory of fuzzy sets, which generalizes the metric spaces. Later on, George and Veeramani [4] presented slight modification on the definition of fuzzy metric spaces initiated by the respective authors by obtaining Hausdorff topology on the same setting. The following definition of fuzzy metric spaces is due to Kramosil and Michalek [14].

Definition 2. An ordered triple $(X, M, *)$ is called a fuzzy metric space if $\mathbf{X}$ is a non-empty set, $\mathbf{M}$ is a fuzzy set on $\mathbf{X}^{2} \times(0, \infty)$ and $*$ is a continuous $\mathrm{t}$-norm satisfying the following conditions for all $\mu, \nu, \xi \in \mathrm{X}$ and $t, s>0$ :

(1) $\mathrm{M}(\mu, \nu, 0)=0$;

(2) $\mathrm{M}(\mu, \nu, t)=1$ if and only if $\mu=\nu$;

(3) $\mathrm{M}(\mu, \nu, t)=\mathrm{M}(\nu, \mu, t)$;

(4) $\mathrm{M}(\mu, \xi, t+s) \geq \mathrm{M}(\mu, \nu, t) * \mathrm{M}(\nu, \xi, s)$;

(5) $\mathrm{M}(\mu, \nu,):.(0, \infty) \longrightarrow(0,1]$ is left continuous.

Definition 3. An ordered triple $(X, M, *)$ is called a fuzzy metric space (in George and Veeramani sense), if in the above Definition conditon (1) and (5) are replaced respectively, with (1') and (5') below:

(1') $\mathrm{M}(\mu, \nu, t)>0$

(5') $\mathrm{M}(\mu, \nu,):.(0, \infty) \longrightarrow(0,1]$ is left continuous.

If condition (4) is replaced by condition

(4') $\mathrm{M}(\mu, \xi, t) \geq \mathrm{M}(\mu, \nu, t) * \mathrm{M}(\nu, \xi, t)$;

then $(\mathrm{X}, \mathrm{M}, *)$ is called a strong fuzzy metric space [7].

Example 2. Let $\mathrm{X}=\mathbb{R}$ and $\mathrm{M}(\mu, \nu, t)=\left[\exp \left(\frac{|\mu-\nu|}{t}\right)\right]^{-1}$. Define continuous t-norm as $\rho * \sigma=\rho \sigma$ for all $\mu, \nu \in \mathbf{X}$ and $t \in(0, \infty)$. Then $\mathrm{M}(\mu, \nu, t)$ is a fuzzy metric space.

Definition 4 ([4]). (i) Let $(\mathrm{X}, \mathrm{M}, *)$ be a fuzzy metric space. A sequence $\left\{\mu_{n}\right\}$ is said to converge to $\mu$ in $\mathrm{X}$ if and only if $\lim _{n \rightarrow \infty} \mathrm{M}\left(\mu_{n}, \mu, t\right)=1$ for all $t>0$, i.e. for each $r \in(0,1)$ and $t>0$, there exists $n_{0} \in \mathbb{N}$ such that $\mathrm{M}\left(\mu_{n}, \mu, t\right)>1-r$, for all $n \geq n_{0}$.

(ii) A sequence $\left\{\mu_{n}\right\}$ in a fuzzy metric space $(\mathrm{X}, \mathrm{M}, *)$ is a $\mathrm{M}$-Cauchy sequence if and only if for each $\epsilon>0, t>0$ there exists $n_{0} \in \mathbb{N}$ such that $\mathrm{M}\left(\mu_{n}, \mu_{m}, t\right)>1-\epsilon$ for all $n, m>n_{0}$. On other hand, $\left\{\mu_{n}\right\}$ is called a Cauchy sequence if $\lim _{n \rightarrow \infty} \mathrm{M}\left(\mu_{n}, \mu_{n+m}, t\right)=1$, for all $m \in \mathbb{N}$ and $t>0$.

(iii) A fuzzy metric space $(X, M, *)$ is complete if every Cauchy sequence in $\mathrm{X}$ is convergent to some $\mu \in \mathrm{X}$. 
Many interesting results have been proved by eminent mathematicians, leading to extensive theories on fuzzy metric setting (see, for instance, [5-35]). In 1912, Shen et al. [23] established an important fixed point result using the concept of altering distance function in fuzzy metric spaces. However, the idea of an altering distance was earlier established and utilized by Khan et al. [12] in the setting of metric spaces.

Nowadays, several authors are adopting the concept of binary relation for generalizing, weakening the existing fixed point results or obtaining new results in the metrical fixed point theory. The notion of continuity and completeness are also weakened with the help of binary relation and developed several interesting results in the setting of relational metric spaces (see, for instance, $[1-3,17,21,30])$.

In this article, $\stackrel{R}{R}, \mathbb{N}$ and $\mathbb{R}$ denotes a nonempty binary relation, the set of all natural numbers and the set of all real numbers respectively.

Definition $5([15])$. A subset of $\mathrm{X} \times \mathrm{X}$ (X be a nonempty set) is said to be a binary relation denoted as $\stackrel{\circ}{R}$, if $(\mu, \nu) \in \stackrel{\circ}{R}$ then $\mu$ is related to $\nu$ under $\stackrel{\circ}{R}$.

Definition 6 ([1]). Let $\mathrm{X}$ be a non empty set equipped with a binary relation $\stackrel{\circ}{R}$ and if either $(\mu, \nu) \in \stackrel{\circ}{R}$ or $(\nu, \mu) \in \stackrel{\circ}{R}$ then $\mu$ and $\nu$ are said to be $\stackrel{\circ}{R}$ comparative, denoted as $[\mu, \nu] \in \stackrel{\circ}{R}$.

Definition $7([15])$. Let $X$ be a nonempty set equipped with a binary relation $\stackrel{\circ}{R}$.

1. The inverse relation $\stackrel{\circ}{R}^{-1}$ of $\stackrel{\circ}{R}$ is defined as follows.

$$
\stackrel{\circ}{R}^{-1}=\{(\mu, \nu) \in \mathrm{X} \times \mathrm{X}:(\nu, \mu) \in \stackrel{\circ}{R}\} .
$$

2. The symmetric closure $\stackrel{\circ}{R}^{s}$ of $\stackrel{\circ}{R}$ is defined as $\stackrel{\circ}{R} \cup \stackrel{\circ}{R}^{-1}$ (indeed, $\stackrel{\circ}{R}^{s}$ is the smallest symmetric relation on $\mathrm{X}$ containing $\stackrel{\circ}{R})$.

Definition 8 ([1]). Let $\mathrm{X}$ be a nonempty set equipped with a binary relation $\stackrel{\circ}{R}$. A sequence $\left\{\mu_{n}\right\}_{n \in \mathbb{N} \cup\{0\}}$ is said to be $\stackrel{\circ}{R}$-preserving if $\left(\mu_{n}, \mu_{n+1}\right) \in \stackrel{\circ}{R}$, for all $n \in \mathbb{N} \cup\{0\}$.

Definition $9([1])$. Let $\mathrm{X}$ be a nonempty set equipped with a binary relation $\stackrel{\circ}{R}$ and $\mathrm{G}$ a self-mapping on $\mathrm{X} . \stackrel{\circ}{R}$ is said to be $\mathrm{G}$-closed if for any $\mu, \nu \in$ $\mathrm{X},(\mu, \nu) \in \stackrel{\circ}{R} \Longrightarrow(\mathrm{G} x, \mathrm{G} y) \in \stackrel{\circ}{R}$.

Proposition 2 ([1]). Let $\stackrel{\circ}{R}$ be a binary relation on a nonempty set $\mathrm{X}$ and $\mathrm{G}$ a self mapping on $\mathrm{X}$. If $\stackrel{\circ}{R}$ is $\mathrm{G}$-closed, then $\stackrel{\circ}{R}^{\text {s }}$ is also $\mathrm{G}$-closed.

Proposition 3 ([1]). Let $\stackrel{\circ}{R}$ be a binary relation on a nonempty set $\mathrm{X}$ and $\mathrm{G}$ a self mapping on $\mathrm{X}$. If $\stackrel{\circ}{R}$ is $\mathrm{G}$-closed, then $\stackrel{\circ}{R}$ is also $\mathrm{G}^{n}$-Closed $\left(\mathrm{G}^{n}\right.$ is $n^{\text {th }}$ iterates of $\mathrm{G}$ ) for all $n \in \mathbb{N} \cup\{0\}$.

Definition 10 ([2]). Let $\mathrm{X}$ be a nonempty set equipped with a binary relation $\stackrel{\circ}{R}$. If for each pair $\mu, \nu \in E \subseteq \mathrm{X}$, there exist a path from $\mu$ to $\nu$ in $\stackrel{\circ}{R}$ then $\mathrm{E}$ is said to be $\stackrel{\circ}{R}$-connected. 
Definition $11([18])$. Let $X$ be a nonempty set equipped with a binary relation $\stackrel{\circ}{R}$. If for each pair $\mu, \nu \in E \subseteq \mathrm{X}$, there exists $\xi \in \mathrm{X}$ such that $(\mu, \xi) \in \stackrel{\circ}{R}$ and $(\xi, \nu) \in \stackrel{\circ}{R}$ then $\mathrm{E}$ is said to be $\stackrel{\circ}{R}$-directed.

Definition $12([2])$. Let $X$ be a nonempty set equipped with a binary relation $\mathbf{R}$ and $\mathbf{G}$ a self-mapping on $\mathbf{X}$. If for any $\mu, \nu, \xi \in \mathbf{X},(\mathrm{G} x, \mathrm{G} z),(\mathrm{G} z, \mathrm{G} y) \in$ $\stackrel{\circ}{R} \Longrightarrow(\mathrm{G} x, \mathrm{G} y) \in \stackrel{\circ}{R}$ then the binary relation $\stackrel{\circ}{R}$ is said to be $\mathrm{G}$-transitive.

Inspired by Turinici (see, $[26,27]$ ), the notion of transitivity was localized by Alam and Imdad [2] as:

Definition 13. Let $\mathrm{X}$ be a nonempty set and $\stackrel{\circ}{R}$ a binary relation on $\mathrm{X}$. Then $\stackrel{\circ}{R}$ is called locally transitive if for each (effectively) $\stackrel{\circ}{R}$-preserving sequence $\left\{\mu_{n}\right\} \subset \mathrm{X}$ (with range $F:=\left\{\left\{\mu_{n}\right\}: n \in \mathbb{N} \cup\{0\}\right\}$ ), the binary relation $\left.\stackrel{\circ}{R}\right|_{F}$ is transitive (throughout the paper, $\left.\stackrel{\circ}{R}\right|_{F}$ denotes the restriction of $\stackrel{\circ}{R}$ to $\mathrm{F}$ ).

Definition $14([2])$. Let $\mathrm{X}$ be a nonempty set and $\stackrel{R}{R}$ be a binary relation on $\mathrm{X}$. Then $\stackrel{R}{R}$ is called locally G-transitive(where $\mathrm{G}$ is a self-mapping on $\mathrm{X}$ ) if for each (effectively) $\stackrel{\circ}{R}$-preserving sequence $\left\{\mu_{n}\right\} \subset \mathrm{G}(\mathrm{X})$ (with range $\left.F:=\left\{\left\{\mu_{n}\right\}: n \in \mathbb{N} \cup\{0\}\right\}\right)$, the binary relation $\left.\stackrel{\circ}{R}\right|_{F}$ is transitive.

Remark 1. The notions of G-transitivity and local transitivity are independent to each other and relatively weaker than the notion of transitivity. In order to make them compatible the notion of locally G-transitivity was introduced and this notion is weaker than the above notions.

Definition 15 ([13]). Let $X$ be a nonempty set equipped with a binary relation $\stackrel{\circ}{R}$. For $\mu, \nu \in \mathrm{X}$, a path of length $m(m \in \mathbb{N})$ in $\mathbb{R}$ from $\mu$ to $\nu$ is a finite sequence $\left\{\zeta_{0}, \zeta_{1}, \zeta_{2}, \ldots, \zeta_{k}\right\} \subset \mathrm{X}$ satisfying the following conditions:

(i) $\zeta_{0}=\mu$ and $\zeta_{k}=\nu$,

(ii) $\left(\zeta_{i}, \zeta_{i+1}\right) \in \stackrel{\circ}{R}$, for each $i(0 \leq i \leq m-1)$.

Notice that the path of length $m$ contains $m+1$ elements of $\mathbf{X}$, although they are not neccessarily distinct.

Given a self-mapping $\mathrm{G}$ and a binary relation $\stackrel{\circ}{R}$ on a nonempty set $\mathrm{X}$, we use the notion $\mathrm{X}(\mathrm{G}, \stackrel{\circ}{R}):=\{\mu \in \mathrm{X}:(\mu, \mathrm{G} \mu) \in \stackrel{\circ}{R}\}$.

Definition $16([23])$. A function $\chi:[0,1] \rightarrow[0,1]$ is said to be an altering distance function if the following properties hold:

(i) $\chi$ is left continuous and strictly decreasing function,

(ii) $\chi(t)=0$ if and only if $t=1$.

It is clear that $\lim _{t \rightarrow 1^{-}} \chi(t)=\chi(1)=0$.

Our objective in this paper, is to present relation-theoretic variant of the fixed point theorem due to Shen et al. [23] in the framework of fuzzy metric and strong fuzzy metric spaces. In obtaining the relational variant of 
the mentioned fixed point results, we introduce the relation theoretic fuzzy analogues of some standard metric notions and utilize the same to prove our fixed point results. As an application of our results, we present the existence and uniqueness of the solution of ordinary differential equation. Moreover, we also furnish an example to support our main results.

\section{MAIN RESUlts}

Definition 17. Let $(X, M, *)$ be a fuzzy metric space endowed with a binary relation $\stackrel{\circ}{R}$. A mapping $\mathrm{G}: \mathrm{X} \rightarrow \mathrm{X}$ is called $\stackrel{\circ}{R}$-continuous at a point $\mu \in \mathrm{X}$ if, for any $\stackrel{\circ}{R}$-preserving sequence $\mu_{n}$ in $\mathrm{X}$ such that $\lim _{n \rightarrow \infty} \mathrm{M}\left(\mu_{n}, \mu, t\right)=1$, we have $\lim _{n \rightarrow \infty} \mathrm{M}\left(\mathrm{G} \mu_{n}, \mathrm{G} \mu, t\right)=1$, for $t>0$. Moreover, $\mathrm{G}$ is called $\stackrel{\circ}{R}$-continuous if it is $\stackrel{\circ}{R}$-continuous at every point of $\mathrm{X}$.

In the fuzzy metric spaces, every continuous mapping is $\stackrel{\circ}{R}$-continuous for any binary relation $\stackrel{\circ}{R}$. Particularly, under the universal relation the notion of $\stackrel{\circ}{R}$-continuity coincides with usual continuity.

Definition 18. Let $(X, M, *)$ be a fuzzy metric space endowed with a binary relation $\stackrel{\circ}{R}$. The space $(\mathrm{X}, \mathrm{M}, *)$ is called $\stackrel{\circ}{R}$-Complete if every $\stackrel{\circ}{R}$-preserving Cauchy sequence in $\mathrm{X}$ converges to some $\mu \in \mathrm{X}$.

Clearly, for any binary relation $\stackrel{\circ}{R}$, every complete fuzzy metric space is $\stackrel{\circ}{R}$-complete. Particularly, under the universal relation the notion of $\stackrel{R}{R}$ completeness coincides with the usual completeness.

Remark 2. In example 3 of this paper, we see that $\mathrm{G}$ is $\stackrel{\circ}{R}$-continuous but not continuous and the space $(\mathrm{X}, \mathrm{M}, *)$ is $\stackrel{\circ}{R}$-complete but not complete fuzzy metric space.

Definition 19. Let $(X, M, *)$ be a fuzzy metric space endowed with a binary relation $\stackrel{\circ}{R}$. The space $(\mathrm{X}, \mathrm{M}, *)$ is called to be $\stackrel{\circ}{R}$-sequentially compact, if for every $\stackrel{\circ}{R}$-preserving sequence $\left\{\mu_{n}\right\}$ in $\mathrm{X}$ such that $\lim _{n \rightarrow \infty} \mathrm{M}\left(\mu_{n+1}, \mu_{n}, t\right)=1$, for $t>0$, there exists a convergent subsequence $\left\{\mu_{n_{k}}\right\}_{k \in \mathbb{N}}$ of $\left\{\mu_{n}\right\}$, which converges to $\mu \in \mathrm{X}$ with $\left[\mu_{n_{k}}, \mu\right] \in \stackrel{\circ}{R}$ for all $k \in \mathbb{N} \cup\{0\}$.

Definition 20. Let $(X, M, *)$ be a fuzzy metric space endowed with a binary relation $\stackrel{\circ}{R}$. We say that $(\mathrm{X}, \mathrm{M}, \stackrel{\circ}{R})$ is regular, if for any $\stackrel{\circ}{R}$-preserving sequence $\left\{\mu_{n}\right\}$ in $\mathrm{X}$ such that $\lim _{n \rightarrow \infty} \mathrm{M}\left(\mu_{n}, \mu, t\right)=1$, for $t>0$, there exists a subsequence $\left\{\mu_{n_{k}}\right\}_{k \in \mathbb{N}}$ of $\left\{\mu_{n}\right\}$ with $\left[\mu_{n_{k}}, x\right] \in \stackrel{\circ}{R}$, for all $k \in \mathbb{N} \cup\{0\}$.

Proposition 4. Let $(\mathrm{X}, \mathrm{M}, *)$ be a fuzzy metric space endowed with a binary relation $\stackrel{\circ}{R}$. Let $\mathrm{G}$ be a self-mapping on $\mathrm{X}$ and $\eta$ be a function from $(0, \infty)$ into $(0,1)$ and $\chi$ be an altering distance function, then the following contractive conditions are equivalent: 
(i) $\chi(\mathrm{M}(\mathrm{G} \mu, \mathrm{G} \nu, t)) \leq \eta(t) \cdot \chi(\mathrm{M}(\mu, \nu, t))$, for all $\mu, \nu \in \mathrm{X}, \mu \neq \nu, t>0$, with $(\mu, \nu) \in \stackrel{\circ}{R}$;

(ii) $\chi(\mathrm{M}(\mathrm{G} \mu, \mathrm{G} \nu, t)) \leq \eta(t) \cdot \chi(\mathrm{M}(\mu, \nu, t))$, for all $\mu, \nu \in \mathrm{X}, \mu \neq \nu, t>0$, with $[\mu, \nu] \in \stackrel{\circ}{R}$.

Proof. Proof of (ii) implies (i) is trivial. Now suppose (i) holds, using the definition of fuzzy metric spaces, $(\mu, \nu) \in \stackrel{\circ}{R}$, we have

$\chi(\mathrm{M}(\mathrm{G} \nu, \mathrm{G} \mu, t))=\chi(\mathrm{M}(\mathrm{G} \mu, \mathrm{G} \nu, t)) \leq \eta(t) \cdot \chi(\mathrm{M}(\mu, \nu, t))=\eta(t) \cdot \chi(\mathrm{M}(\nu, \mu, t))$, for all $\mu, \nu \in \mathrm{X}, \mu \neq \nu, t>0$, with $(\nu, \mu) \in \stackrel{\circ}{R}$.

Hence, the above inequality shows that (i) implies (ii).

Theorem 1. Let $(\mathrm{X}, \mathrm{M}, *)$ be a fuzzy metric space endowed with a binary relation $\stackrel{\circ}{R}$. Let $\mathrm{G}$ be a self-mapping on $\mathrm{X}$. Suppose that the following conditions hold:

(i) $\mathrm{X}(\mathrm{G}, \stackrel{\circ}{R})$ is nonempty,

(ii) $\stackrel{\circ}{R}$ is G-closed,

(iii) there exists $\eta:(0, \infty) \rightarrow(0,1)$ and an altering distance function $\chi$ such that

$$
\chi(\mathrm{M}(\mathrm{G} \mu, \mathrm{G} \nu, t)) \leq \eta(t) \cdot \chi(\mathrm{M}(\mu, \nu, t)),
$$

for all $\mu, \nu \in \mathrm{X}, \mu \neq \nu, t>0$, with $(\mu, \nu) \in \stackrel{\circ}{R}$,

(iv) $(\mathrm{X}, \mathrm{M}, *)$ is $\stackrel{\circ}{R}$-sequentially compact.

Or

(v) (a) $(\mathrm{X}, \mathrm{M}, *)$ is a complete strong fuzzy metric space and $*$ is a $t$ norm of H-type,

(b) $(\mathrm{X}, \mathrm{M}, \stackrel{\circ}{R})$ is regular.

Then $\mathrm{G}$ has a fixed point.

Proof. Let $\mu_{0} \in \mathrm{X}(\mathrm{G}, \stackrel{\circ}{R})$ be an arbitrary element. We can define a sequence $\left\{\mu_{n}\right\}$ by Picard iteration, i.e., $\mu_{n+1}=\mathrm{G} \mu_{n}$, for all $n \in \mathbb{N} \cup\{0\}$. Using condition (ii), as $\left(\mu_{0}, B \mu_{0}\right) \in \stackrel{\circ}{R}$, we get

$$
\left(\mathrm{G} \mu_{0}, \mathrm{G}^{2} \mu_{0}\right),\left(\mathrm{G}^{2} \mu_{0}, \mathrm{G}^{3} \mu_{0}\right), \ldots,\left(\mathrm{G}^{n} \mu_{0}, \mathrm{G}^{n+1} \mu_{0}\right), \ldots \in \stackrel{\circ}{R},
$$

in such way that $\left(\mu_{n}, \mu_{n+1}\right) \in \stackrel{\circ}{R}$, for all $n \in \mathbb{N} \cup\{0\}$ and the sequence $\left\{\mu_{n}\right\}$ is a $\stackrel{\circ}{R}$ - preserving sequence.

Denote $\mathrm{M}_{n}(t)=\mathrm{M}\left(\mu_{n}, \mu_{n+1}, t\right)$. As $\left(\mu_{n}, \mu_{n+1}\right) \in \stackrel{\circ}{R}$, from condition (iii), for every $t>0$, we get

$$
\begin{aligned}
\chi\left(\mathrm{M}_{n}(t)\right) & =\chi\left(\mathrm{M}\left(\mu_{n}, \mu_{n+1}, t\right)\right) \\
& =\chi\left(\mathrm{M}\left(\mathrm{G} \mu_{n-1}, \mathrm{G} \mu_{n}, t\right)\right) \leq \eta(t) \cdot \chi\left(\mathrm{M}\left(\mu_{n-1}, \mu_{n}, t\right)\right) \\
& =\eta(t) \cdot \chi\left(\mathrm{M}_{n-1}(t)\right)<\chi\left(\mathrm{M}_{n-1}(t)\right) .
\end{aligned}
$$

$\chi$ is strictly decreasing, so that $\mathrm{M}_{n}(t)>\mathrm{M}_{n-1}(t)$, i.e., $\mathrm{M}\left(\mu_{n}, \mu_{n+1}, t\right)>$ $\mathrm{M}\left(\mu_{n-1}, \mu_{n}, t\right)$, for $t>0$. 
Hence the sequence $\left\{\mathrm{M}_{n}(t)\right\}$ is a non-decreasing and bounded sequence for $t>0$, so there exists $\gamma:(0, \infty) \rightarrow[0.1]$ such that

$$
\lim _{n \rightarrow \infty} \mathrm{M}_{n}(t)=\lim _{n \rightarrow \infty} \mathrm{M}\left(\mu_{n}, \mu_{n+1}, t\right)=\gamma(t), t>0 .
$$

From (1), (2) and letting $n \rightarrow \infty$ for $t>0$, we have

$$
\chi(\gamma(t)) \leq \eta(t) \cdot \chi(\gamma(t))<\chi(\gamma(t)),
$$

which implies that $\gamma(t) \equiv 1$, for $t>0$, i.e.,

$$
\lim _{n \rightarrow \infty} \mathrm{M}\left(\mu_{n}, \mu_{n+1}, t\right)=1 \text {. }
$$

Now suppose that the (v) (a) holds. As $*$ is a t-norm of H-type, using (2) and proposition 1 , there exists $n_{1} \in \mathbb{N}$ such that

$$
*_{i=n}^{\infty} \mathrm{M}\left(\mu_{i}, \mu_{i+1}, t\right)>1-\epsilon, t>0, n \geq n_{1} .
$$

Since $\left\{*_{i=1}^{n} \mathrm{M}\left(\mu_{i}, \mu_{i+1}, t\right)\right\}_{n \in \mathbb{N}}$ is a non-decreasing sequence and $(\mathrm{X}, \mathrm{M}, *)$ is a strong fuzzy metric space, from (5), we get

(6) $\mathrm{M}\left(\mu_{n+r+1}, \mu_{n}, t\right) \geq *_{i=n}^{n+s} \mathrm{M}\left(\mu_{i}, \mu_{i+1}, t\right)>1-\epsilon, t>0, n \geq n_{1}, r \in \mathbb{N}$.

Hence $\left\{\mu_{n}\right\}$ is a Cauchy sequence and due to completeness of space (X, M,*), there exists $\mu \in \mathbf{X}$ such that

$$
\lim _{n \rightarrow \infty} \mu_{n}=\mu .
$$

Now, we have to show that $\mu$ is the fixed point of the map G. As $\left\{\mu_{n}\right\}$ is an $\stackrel{\circ}{R}$-preserving sequence, by condition (v) (b), we have a subsequence $\left\{\mu_{n_{k}}\right\}_{n \in \mathbb{N}}$ of $\left\{\mu_{n}\right\}$ such that

$$
\left[\mu_{n_{k}}, \mu\right] \in \stackrel{\circ}{R} \text { for all } k \in \mathbb{N},
$$

by condition (iii) and Proposition 4 , for $t>0$, we have

$$
\begin{array}{r}
0 \leq \chi\left(\mathrm{M}\left(\mu_{n_{k+1}}, \mathrm{G} \mu, t\right)\right)=\chi\left(\mathrm{M}\left(\mathrm{G} \mu_{n_{k}}, \mathrm{G} \mu, t\right)\right) \\
\leq \eta(t) \cdot \chi\left(\mathrm{M}\left(\mu_{n_{k}}, \mu, t\right)\right),
\end{array}
$$

as $n \rightarrow \infty$, we have

$$
\chi(\mathrm{M}(\mu, \mathrm{G} \mu, t)) \leq \eta(t) \cdot \chi(1),
$$

i.e., $\chi(\mathrm{M}(\mathrm{G} \mu, \mu, t))=0$, which implies that $\mathrm{M}(\mathrm{G} \mu, \mu, t)=1$. Hence $\mu$ is a fixed point of $\mathrm{G}$.

Suppose that condition (iv) holds. As $\left\{\mu_{n}\right\}$ is a $\stackrel{\circ}{R}$-preserving sequence and from (4) we have a convergent subsequence $\left\{\mu_{n_{k}}\right\}$ which converges to $\mu$ and $\left[\mu_{n_{k}}, \mu\right] \in \stackrel{\circ}{R}$, for all $k \in \mathbb{N} \cup\{0\}$. Now, we only have to prove that $\mu$ is a fixed point of $\mathrm{G}$, which is similar to the proof given above.

Remark 3. In the above Theorem 1 , we introduce the $\stackrel{\circ}{R}$-sequentially compact property to omit the completeness property of fuzzy metric spaces and in this part of the proof of this Theorem, we do not require to show that the $\stackrel{\circ}{R}$-preserving sequence $\left\{\mu_{n}\right\}$ is a Cauchy sequence. 
Theorem 2. Let $(\mathrm{X}, \mathrm{M}, *)$ be a fuzzy metric space endowed with a binary relation $\stackrel{\circ}{R}$. Let $\mathrm{G}$ be a self-mapping on $\mathrm{X}$. Suppose that the following conditions hold:

(i) $\mathrm{X}(\mathrm{G}, \stackrel{\circ}{R})$ is nonempty,

(ii) $\stackrel{\circ}{R}$ is G-closed,

(iii) $(\mathrm{X}, \mathrm{M}, *)$ is $\stackrel{\circ}{\mathrm{R}}$-complete,

(iv) there exists $\eta:(0, \infty) \rightarrow(0,1)$ and an altering distance function $\chi$ such that

$$
\chi(\mathrm{M}(\mathrm{G} \mu, \mathrm{G} \nu, t)) \leq \eta(t) \cdot \chi(\mathrm{M}(\mu, \nu, t)),
$$

for all $\mu, \nu \in \mathbf{X}, \mu \neq \nu, t>0$, with $(\mu, \nu) \in \stackrel{\circ}{R}$,

(v) $\stackrel{\circ}{R}$ is locally G-transitive,

(vi) (a) G is $\stackrel{\circ}{R}$-continuous, or

(b) $(\mathrm{X}, \mathrm{M}, \stackrel{\circ}{R})$ is regular.

Then $\mathrm{G}$ has a fixed point.

Proof. From the conditions (i), (ii) and (iv), we get an $\stackrel{\circ}{R}$-preserving sequence $\left\{\mu_{n}\right\}_{n \in \mathbb{N} \cup\{0\}}$ such that

$$
\lim _{n \rightarrow \infty} \mathrm{M}\left(\mu_{n}, \mu_{n+1}, t\right)=1, t>0 .
$$

The proof of above is analogous to the proof given in Theorem 1.

Now, we show that the sequence $\left\{\mu_{n}\right\}$ is a Cauchy sequence. On the contrary, suppose that $\left\{\mu_{n}\right\}$ is not a Cauchy sequence, then there exist $0<\epsilon<1, t>0$ and two subsequences $\left\{\mu_{m_{j}}\right\}$ and $\left\{\mu_{n_{j}}\right\}$ of sequence $\left\{\mu_{n}\right\}$ with $m_{j}>n_{j} \geq j$, for $j \in \mathbb{N} \cup\{0\}$, such that

$\mathrm{M}\left(\mu_{m_{j}}, \mu_{n_{j}}, t\right) \leq 1-\epsilon, \mathrm{M}\left(\mu_{m_{j-1}}, \mu_{n_{j-1}}, t\right)=1-\epsilon$ and $\mathrm{M}\left(\mu_{m_{j-1}}, \mu_{n_{j}}, t\right)=1-\epsilon$.

From definition of fuzzy metric space and (10), we have

$$
\begin{array}{r}
1-\epsilon \geq \mathrm{M}\left(\mu_{m_{j}}, \mu_{n_{j}}, t\right) \geq \mathrm{M}\left(\mu_{m_{j-1}}, \mu_{m_{j}}, t\right) * \mathrm{M}\left(\mu_{m_{j-1}}, \mu_{n_{j}}, t\right) \\
=\mathrm{M}_{m_{j}}(t) * \mathrm{M}\left(\mu_{m_{j-1}}, \mu_{n_{j}}, t\right) .
\end{array}
$$

Since $\lim _{j \rightarrow \infty} \mathrm{M}_{m_{j}}(t)=1$, for $t>0$, from (10) and (11), we have

$$
\lim _{j \rightarrow \infty} \mathrm{M}\left(\mu_{m_{j}}, \mu_{n_{j}}, t\right) \rightarrow 1-\epsilon, t>0 .
$$

Moreover, as $\left\{\mu_{n}\right\}$ is an $\stackrel{\circ}{R}$-preserving sequence and $\left\{\mu_{n}\right\}$ is based on Picard iteration, so by condition (v), we have $\left(\mu_{m_{j}}, \mu_{n_{j}}\right) \in \stackrel{\circ}{R}$, for every $j$. Now in the light of condition (iv), for each $t>0$, we get

$$
\begin{aligned}
\chi\left(\mathrm{M}\left(\mu_{m_{j}}, \mu_{n_{j}}, t\right)\right) & =\chi\left(\mathrm{M}\left(\mathrm{G} \mu_{m_{j-1}}, \mathrm{G} \mu_{n_{j-1}}, t\right)\right) \\
& \leq \eta(t) \cdot \chi\left(\mathrm{M}\left(\mu_{m_{j-1}}, \mu_{n_{j-1}}, t\right)\right)<\chi\left(\mathrm{M}\left(\mu_{m_{j-1}}, \mu_{n_{j-1}}, t\right)\right) .
\end{aligned}
$$


Using definition of $\chi, \mathrm{M}\left(\mu_{m_{j}}, \mu_{n_{j}}, t\right)>\mathrm{M}\left(\mu_{m_{j-1}}, \mu_{n_{j-1}}, t\right)$, for each $j \in \mathbb{N}$, $t>0$ and from (10), we get

$$
1-\epsilon \geq \mathrm{M}\left(\mu_{m_{j}}, \mu_{n_{j}}, t\right)>\mathrm{M}\left(\mu_{m_{j-1}}, \mu_{n_{j-1}}, t\right)>1-\epsilon,
$$

which is a contradiction. Hence $\left\{\mu_{n}\right\}$ is a Cauchy sequence. Owing to $\stackrel{\circ}{R}$-completeness of the space $(\mathrm{X}, \mathrm{M}, *)$, we have a point $\mu \in \mathrm{X}$ such that sequence $\left\{\mu_{n}\right\}$ converges to $\mu$.

At last we have to prove that $\mu$ is the fixed point of the map G. By condition (vi) (a), G is $\stackrel{\circ}{R}$-continuous and $\mu_{n}$ is $\stackrel{\circ}{R}$-preserving sequence such that $\lim _{n \rightarrow \infty} \mathrm{M}\left(\mu_{n}, \mu, t\right)=1$, which implies that

$$
\lim _{n \rightarrow \infty} \mathrm{M}\left(\mathrm{G} \mu_{n}, \mathrm{G} \mu, t\right)=1 \text {, for all } t>0 .
$$

Uniqueness of limit implies that $\mathrm{G} \mu=\mu$.

To prove $\mu$ is a fixed point of G, we use condition (vi) (b) and following the lines of proof presented in Theorem 1 .

Corollary 1. Let $(\mathrm{X}, \mathrm{M}, *)$ be a fuzzy metric space endowed with a binary relation $\stackrel{\circ}{R}$. Let $\mathrm{G}$ be a self-mapping on $\mathrm{X}$. Suppose that following conditions hold:

(i) $\mathrm{X}(\mathrm{G}, \stackrel{\circ}{R})$ is nonempty,

(ii) $\stackrel{\circ}{R}$ is G-closed,

(iii) $(\mathrm{X}, \mathrm{M}, *)$ is $\stackrel{\circ}{\mathrm{R}}$-complete,

(iv) there exists an altering distance function $\chi$ such that

$$
\chi(\mathrm{M}(\mathrm{G} \mu, \mathrm{G} \nu, t))<\chi(\mathrm{M}(\mu, \nu, t)),
$$

for all $\mu, \nu \in \mathrm{X}, \mu \neq \nu, t>0$, with $(\mu, \nu) \in \stackrel{\circ}{R}$,

(v) $\stackrel{\circ}{R}$ is locally G-Transitive,

(vi) (a) G is $\stackrel{\circ}{R}$-continuous, or

(b) $(\mathrm{X}, \mathrm{M}, \stackrel{\circ}{R})$ is regular.

Then $\mathrm{G}$ has a fixed point.

Proof. As

$$
\chi(\mathrm{M}(\mathrm{G} \mu, \mathrm{G} \nu, t))<\chi(\mathrm{M}(\mu, \nu, t)),
$$

for all $\mu, \nu \in \mathrm{X}, \mu \neq \nu, t>0$, with $(\mu, \nu) \in \stackrel{\circ}{R}$, then following the lines of the proof of Theorem 2 above we get the result.

Remark 4. Instead of condition (v) in the Theorem 2 and Corollary 1 if we use one of the following conditions, the Theorem will remain true:

(i) $\stackrel{\circ}{R}$ is transitive.

(ii) $\stackrel{\circ}{R}$ is G-transitive.

(iii) $\stackrel{\circ}{R}$ is locally transitive.

Theorem 3. In addition to the hypothesis of Theorem 1, 2 and Corollary 1. Suppose that $\mathrm{G}(\mathrm{X})$ is $\stackrel{\circ}{R}^{s}$-connected. Then $\mathrm{G}$ has a unique fixed point. 
Proof. In the light of Theorem 1 and 2, the set of all fixed points (say $F(\mathrm{G})$ ) of map $\mathrm{G}$ is non empty and suppose $\mu, \nu \in F(\mathrm{G})$, we have

$$
\mathrm{G}^{n}(\mu)=\mu, \mathrm{G}^{n}(\nu)=\nu, \text { for all } n \in \mathbb{N} \cup\{0\} .
$$

As $\mathrm{G}(\mathrm{X})$ is $\stackrel{\circ}{R}^{s}$-connected, so there exists a path (say $\left\{\varsigma_{0}, \varsigma_{1}, \ldots, \varsigma_{m}\right\}$ ) of finite length $\mathrm{m}$ in $\stackrel{\circ}{R}^{s}$ from $\mu$ to $\nu$, such that

$$
\mu=\varsigma_{0}, \nu=\varsigma_{m} \text { and }\left[\varsigma_{i}, \varsigma_{i+1}\right] \in \stackrel{\circ}{R} \text {, for each } 0 \leq i \leq m-1 .
$$

As $\stackrel{\circ}{R}$ is G-closed, from Proposition 2 and 3, we have

$$
\left[\mathrm{G}^{n} \varsigma_{i}, \mathrm{G}^{n} \varsigma_{i+1}\right] \in \stackrel{\circ}{R} \text {, for each } i(0 \leq i \leq m-1) \text { and } n \in \mathbb{N} \cup\{0\} .
$$

Denote $\mathrm{M}_{n}^{i}(t)=\mathrm{M}\left(\mathrm{G}^{n} \varsigma_{i}, \mathrm{M}^{n} \varsigma_{i+1}, t\right)$, for each $i(0 \leq i \leq m-1), n \in \mathbb{N} \cup\{0\}$ and $t>0$.

We claim that $\lim _{n \rightarrow \infty} \mathrm{M}_{n}^{i}(t)=1$, for all $t>0$. Now, for a fix $i$, we discuss two cases.

Firstly, suppose that $\mathrm{M}_{n_{0}}^{i}(t)=\mathrm{M}\left(\mathrm{G}^{n_{0}} \varsigma_{i}, \mathrm{G}^{n_{0}} \varsigma_{i+1}, t\right)=1$, for some $n_{0} \in$ $\mathbb{N}_{0}$ and for all $t>0$, i.e. $\mathrm{G}^{n_{0}} \varsigma_{i}=\mathrm{G}^{n_{0}} \varsigma_{i+1}$, which implies that $\mathrm{G}^{n_{0}+1} \varsigma_{i}=$ $\mathrm{G}^{n_{0}+1} \varsigma_{i+1}$, i.e., $\mathrm{M}_{n_{0}+1}^{i}(t)=\mathrm{M}\left(\mathrm{G}^{n_{0}+1} \varsigma_{i}, \mathrm{G}^{n_{0}+1} \varsigma_{i+1}, t\right)=1$, for all $t>0$. Thus by induction, we have $\mathrm{M}_{n_{0}}^{i}(t)=1$, for all $n \geq n_{0}$ and $t>0$, therefore

$$
\lim _{n \rightarrow \infty} \mathrm{M}_{n}^{i}(t)=\lim _{n \rightarrow \infty} \mathrm{M}\left(\mathrm{G}^{n} \varsigma_{i}, \mathrm{G}^{n} \varsigma_{i+1}, t\right)=1, t>0 .
$$

Secondly, suppose that $\mathrm{M}_{n}^{i}(t)<1$, for all $t>0$. Using (15), Proposition 4 and contractive condition, we have

$$
\begin{aligned}
\chi\left(\mathrm{M}_{n+1}^{i}(t)\right) & =\chi\left(\mathrm{M}\left(\mathrm{G}^{n+1}{ }_{\varsigma_{i}}, \mathrm{G}^{n+1}{ }_{\varsigma_{i+1}}, t\right)\right) \\
& \leq \eta(t) \cdot \chi\left(\mathrm{M}\left(\mathrm{G}^{n} \varsigma_{i}, \mathrm{G}^{n}{ }_{\varsigma_{i+1}}, t\right)\right) \\
& =\eta(t) \cdot \chi\left(\mathrm{M}_{n}^{i}(t)\right)<\chi\left(\mathrm{M}_{n}^{i}(t)\right), t>0 .
\end{aligned}
$$

As $\chi$ is strictly decreasing function, so $\mathrm{M}_{n+1}^{i}(t)>\mathrm{M}_{n}^{i}(t)$ for all $t>0$.

Hence the sequence $\left\{\mathrm{M}_{n}^{i}(t)\right\}$ is non-decreasing and bounded for all $t>0$, for each $i(0 \leq i \leq m-1)$, so there exists $a:(0, \infty) \rightarrow[0,1]$ such that

$$
\lim _{n \rightarrow \infty} \mathrm{M}_{n}^{i}(t)=\lim _{n \rightarrow \infty} \mathrm{M}\left(\mathrm{G}^{n} \varsigma_{i}, \mathrm{G}^{n} \varsigma_{i+1}, t\right)=a(t) \text {, for all } t>0 .
$$

From (17) and (18), we have

$$
\chi(a(t)) \leq \eta(t) \cdot \chi(a(t))<\chi(a(t)), t>0 .
$$

From the definition of $\chi$, we get $a(t)=1$. Thus, in both cases,

$$
\lim _{n \rightarrow \infty} \mathrm{M}_{n}^{i}(t)=\lim _{n \rightarrow \infty} \mathrm{M}\left(\mathrm{G}^{n} \varsigma_{i}, \mathrm{G}^{n} \varsigma_{i+1}, t\right)=1 \text {, for all } t>0 .
$$

Now, from (13), (14), (19), definition of fuzzy metric spaces and $n \rightarrow \infty$, we get 


$$
\begin{aligned}
& \mathrm{M}(\mu, \nu, t)= \mathrm{M}\left(\mathrm{G}^{n} \varsigma_{0}, \mathrm{G}^{n} \varsigma_{m}, t\right) \\
& \geq \mathrm{M}\left(\mathrm{G}^{n} \varsigma_{0}, \mathrm{G}^{n} \varsigma_{1}, \frac{t}{m}\right) * \mathrm{M}\left(\mathrm{G}^{n} \varsigma_{1}, \mathrm{G}^{n} \varsigma_{2}, \frac{t}{m}\right) \\
& \quad * \cdots * \mathrm{M}\left(\mathrm{G}^{n} \varsigma_{m-1}, \mathrm{G}^{n} \varsigma_{m}, \frac{t}{m}\right) \\
&=1 .
\end{aligned}
$$

Hence $\mu=\nu$.

Remark 5. Instead of the condition " $\mathrm{G}(\mathrm{X})$ is $\stackrel{\circ}{R}^{s}$-connected" in the hypothesis of Theorem 3, if we use one of the following conditions, the Theorem will remains true:

(i) $\left.\stackrel{\circ}{R}\right|_{\mathrm{G}(\mathrm{X})}$ is complete,

(ii) $\mathrm{G}(\mathrm{X})$ is $\stackrel{\circ}{R}^{s}$-directed.

If conditions (i) and (ii) hold, then for each $\mu, \nu \in F(\mathrm{G})$, there will exist path of length 1 and 2 respectively on $\stackrel{\circ}{R}^{s}$ from $\mu$ to $\nu$. Hence $\mathrm{G}(\mathrm{X})$ is $\stackrel{\circ}{R}^{s}$-connected. By Theorem 3 the proof is immediate.

Example 3. Let $X=\mathbb{R}^{+}$be a set and $(X, M, *)$ a fuzzy metric space, where $*$ is a continuous t-norm

$$
\mathrm{M}(\mu, \nu, t)=\frac{\min \{\mu, \nu\}+t}{\max \{\mu, \nu\}+t}, \text { for all } \mu, \nu \in \mathrm{X} \text {. }
$$

Define a binary relation $\stackrel{\circ}{R}$ on set $\mathrm{X}$ as

$$
\stackrel{\circ}{R}=\{(1,2),(1,3),(1,4),(1,5),(2,1),(2,3),(2,4),(2,5),(3,4),(5,1)\}
$$

and a mapping $\mathrm{G}: \mathrm{X} \rightarrow \mathrm{X}$ such that

$$
\mathrm{G}(\mu)= \begin{cases}2, & \text { if } \mu \in(0,4] \\ 1, & \text { otherwise }\end{cases}
$$

(i) $\mathrm{X}(\mathrm{G}, \stackrel{\circ}{R})$ is non-empty, because $\mathrm{X}(\mathrm{G}, \stackrel{\circ}{R})=\{1,5\}$.

(ii) As for all $(\mu, \nu) \in \stackrel{\circ}{R},(\mathrm{G}(\mu), \mathrm{G}(\nu)) \in\{(1,2),(2,1)\}$ which implies that $\stackrel{\circ}{R}$ is G-closed.

(iii) Suppose that $\left\{\mu_{n}\right\}$ be any $\stackrel{\circ}{R}$-preserving sequence, we know that $\left\{\mu_{n}\right\} \in\{1,2\}$ for every $n \in \mathbb{N}$, which implies that the space $(\mathrm{X}, \mathrm{M}, *)$ is $\stackrel{\circ}{R}$-complete. Notice that the space $(\mathrm{X}, \mathrm{M}, *)$ is not complete.

(iv) Define an altering function $\chi:[0,1] \rightarrow[0,1]$ such that

$$
\chi(a)= \begin{cases}1-a, & \text { if } a \in(0,1), \\ 0, & \text { if } a=1, \\ 1, & \text { if } a=0\end{cases}
$$


and $\eta(t):(0, \infty) \rightarrow(0,1)$ such that $\eta(t)=\frac{9}{10}$, for all $t \in(0, \infty)$.

We have

$$
1-\frac{\min \{\mathrm{G} \mu, \mathrm{G} \nu\}+t}{\max \{\mathrm{G} \mu, \mathrm{G} \nu\}+t} \leq \frac{9}{10} .\left(1-\frac{\min \{\mu, \nu\}+t}{\max \{\mu, \nu\}+t}\right),
$$

for all $(\mu, \nu) \in \stackrel{\circ}{R}$ and $t>0$.

(v) For any $\stackrel{\circ}{R}$-preserving sequence, $\mu_{n} \in\{1,2\}$, range $F=\{1,2\}$ then $\left.\stackrel{\circ}{R}\right|_{F}=\{(1,2)\}$. Hence $\stackrel{\circ}{R}$ is locally G-transitive.

Whereas, $\{(\mathrm{G}(5), \mathrm{G}(1)),(\mathrm{G}(1), \mathrm{G}(5))\} \in \stackrel{\circ}{R}$ but $(\mathrm{G}(5), \mathrm{G}(5)) \notin \stackrel{\circ}{R}$, so $\stackrel{\circ}{R}$ is neither $\mathrm{G}$-transitive nor transitive.

(vi) Conditions (vi) (a) and (b) of Theorem 2 are satisfied, one can easily prove these conditions.

Hence the hypothesis of Theorem 2 is satisfied. So, the mapping $B$ has a fixed point, that is, 2. As $\mathrm{G}(\mathrm{X})$ is $\stackrel{\circ}{R}^{s}$-connected. So, from Theorem 3 the mapping $G$ has a unique fixed pint.

In the light of above non-trivial example, we generalize Theorem 3.1 of paper [23]. We also notice that:

(i) In Theorem 2 and $3 \stackrel{\circ}{R}$-completeness of the space $(\mathrm{X}, \mathrm{M}, *)$ is used, which is relatively weaker than completeness.

(ii) Since our contractive condition enjoys only on those elements which are related under the underlying binary relation $\stackrel{\circ}{R}$. Thus our contractive condition is a generalized version of any other contraction used in the fuzzy metric setting.

(iii) Instead of binary relation $\stackrel{\circ}{R}$, if we take universal relation, i.e. $\stackrel{\circ}{R}=$ $\mathrm{X} \times \mathrm{X}$ then Theorem 2 and 3 coincides with the Theorem 3.1 of [23].

(iv) Our results also generalize the fixed point result in [25]. Choosing altering distance function $\chi(t)=1-t, \eta(t)=\alpha$, where $\alpha \in(0,1)$, t-norm as $\rho * \sigma=\max \{\rho+\sigma-1,0\}$, for all $\rho, \sigma \in[0,1]$ and binary relation as universal relation, our result coincides with Corollary 3.5 presented in [25].

\section{Application}

In this section, as an application we prove existence and uniqueness of solution for the following ordinary differential equation using Corollary 1 and Theorem 3. Problem:

$$
\zeta^{\prime}(p)=g(p, \zeta(p)), p \in I=[0, l], \zeta(0)=\zeta(l),
$$

where $g: I \times \mathbb{R} \rightarrow \mathbb{R}$ is a continuous function and $l>0$ (see, [16]).

Let $C(I)$ denote the space of all continuous functions defined on I. Now we define $*:[0,1] \times[0,1] \rightarrow[0,1]$ such that $\rho * \sigma=\rho \sigma$ and for $\beta(p), \zeta(p) \in C(I)$, $t>0$,

$$
\mathrm{M}(\beta(p), \zeta(p), t)=e^{-\frac{d(\beta(p), \zeta(p))}{t}},
$$


where $d(\beta(p), \zeta(p))=\sup |\beta(p)-\zeta(p)|$, for $p \in I$. Then $(C(I), \mathbf{M}, *)$ is a fuzzy metric space.

Theorem 4. Considering the Problem (20), suppose that there exists $\vartheta>0$ such that for $(\beta, \zeta) \in \stackrel{\circ}{R}$ with $\beta \leq \zeta$,

$$
0 \leq g(p, \zeta)+\vartheta \zeta-[g(p, \beta)+\vartheta \beta]<\vartheta(\zeta-\beta) .
$$

Then there exists a unique solution of Problem (20).

Proof. Problem (20) can be rewritten as

$$
\zeta^{\prime}(p)+\vartheta \zeta(p)=g(p, \zeta(p))+\vartheta \zeta(p), p \in I=[0, l], \zeta(0)=\zeta(l) .
$$

This corresponds to the Integral equation

$$
\zeta(p)=\int_{0}^{l} \kappa(p, s)[g(s, \zeta(s))+\vartheta \zeta(s)] d s
$$

where

$$
\kappa(p, s)= \begin{cases}\frac{e^{\vartheta(l+s-p)}}{e^{\vartheta l}-1}, & 0 \leq s<p \leq l, \\ \frac{e^{\vartheta(s-p)}}{e^{\vartheta l}-1}, & 0 \leq p<s \leq l .\end{cases}
$$

Define a mapping $\mathrm{G}: C(I) \rightarrow C(I)$ such that

$$
(\mathrm{G} \zeta)(p)=\int_{0}^{l} \kappa(p, s)[g(s, \zeta(s))+\vartheta \zeta(s)] d s
$$

and a binary relation

$$
\stackrel{\circ}{R}=\{(\beta(p), \zeta(p)) \in C(I) \times C(I): \beta(p) \leq \zeta(p) \text { with } \beta(p) \zeta(p) \geq 0 \forall p \in I\} .
$$

(i) For any $\zeta(p) \geq 0, p \in I$, we have $\mathrm{G} \zeta(p) \geq 0$ and $\zeta(p) \leq \mathrm{G} \zeta(p)$, for all $p \in I$, which implies that $(\zeta, \mathrm{G} \zeta) \in \stackrel{\circ}{R}$. Hence $\mathbf{X}(\mathrm{G}, \stackrel{\circ}{R})$ is non-empty.

(ii) Suppose $(\beta, \zeta) \in \stackrel{\circ}{R}$, i.e. $\beta(p) \leq \zeta(p)$, by (22) and for each $p \in I$ we have

$$
g(p, \beta(p))+\vartheta \beta(p)<g(p, \zeta(p))+\vartheta \zeta(p) .
$$

As, $\kappa(p, s)>0,(p, s) \in I \times I$ and by $(25)$, we get $\mathrm{G} \beta(p) \leq \mathrm{G} \zeta(p)$, for every $p \in I$ and obviously, if $\beta(p) \zeta(p) \geq 0$ we will get $\mathrm{G} \beta(p) \mathrm{G} \zeta(p) \geq$ 0 . Hence $(\mathrm{G} \beta, \mathrm{G} \zeta) \in \stackrel{\circ}{R}$, i.e. $\stackrel{\circ}{R}$ is $\mathrm{G}$-closed.

(iii) As $(C(I), \mathrm{M}, *)$ is a complete fuzzy metric space, so it is $\stackrel{\circ}{R}$-complete.

(iv) Now, from $(25)$ and for all $(\beta(p), \zeta(p)) \in \stackrel{\circ}{R}$, we have

$$
\begin{aligned}
& \sup |\mathrm{G} \beta(p)-\mathrm{G} \zeta(p)| \\
& =\sup \left|\int_{0}^{l} \kappa(p, s)[g(s, \zeta(s))+\vartheta \zeta(s)-g(s, \beta(s))-\vartheta \beta(s)] d s\right|, \\
& \quad<\sup \left|\int_{0}^{l} \kappa(p, s) \vartheta(\zeta-\beta) d s\right|,
\end{aligned}
$$




$$
\begin{aligned}
& \leq \vartheta(\sup |\beta-\zeta|)\left(\sup \int_{0}^{l} \kappa(p, s) d s\right) \\
& \leq \vartheta(\sup |\beta-\zeta|)\left\{\sup \frac{1}{\vartheta\left(e^{\vartheta l}-1\right)} \cdot\left(e^{\vartheta l}-1\right)\right\} \\
& =\sup |\beta-\zeta| .
\end{aligned}
$$

Therefore, for all $t>0$, we get

$$
e^{-\frac{d(\mathrm{G} \beta, \mathrm{G} \zeta)}{t}}>e^{-\frac{d(\beta, \zeta)}{t}}
$$

Now, considering an altering distance function $\chi$, we get

$$
\chi(\mathrm{M}(\mathrm{G} \beta, \mathrm{G} \zeta, t))=\chi\left(e^{-\frac{d(\mathrm{G} \beta, \mathrm{G} \zeta)}{t}}\right)<\chi\left(e^{-\frac{d(\beta, \zeta)}{t}}\right)=\chi(K(\beta, \zeta, t)) .
$$

Hence the contractive condition holds for every $(\beta, \zeta) \in \stackrel{\circ}{R}$ and $t>0$.

(v) Choose an $\stackrel{\circ}{R}$-preserving sequence $\left\{\beta_{n}(p)\right\}$, such that it converges to $\beta(p)$. By the defined binary relation $\stackrel{\circ}{R}$, we discuss two cases: $\left(a_{1}\right)$ $\beta_{n}(p) \geq 0$ and $\left(b_{1}\right) \beta_{n}(p) \leq 0$ for all $p \in I$. Considering case $\left(a_{1}\right)$, i.e. $\beta_{n}(p) \geq 0$, a sequence of non-negative real numbers such that $\beta_{n}(p) \rightarrow \beta(p)$, which implies that $\beta(p) \geq 0$, for each $p \in I$. Hence $\left(\beta_{n}(p), \beta(p)\right) \in \stackrel{\circ}{R}$ for all $p \in \stackrel{\circ}{R}$ and $n \in \mathbb{N} \cup\{0\}$. So, condition (vi) (b) of Corollary 1 is satisfied. Also, as $\beta_{n}(p) \geq 0$, then the range set of $\beta_{n}(p)$ (say, E) is a set of non-negative real numbers. So $\left.\stackrel{\circ}{R}\right|_{E}$ is transitive. Hence $\stackrel{\circ}{R}$ is locally G-Transitive.

Since all the conditions of Corollary 1 are satisfied. Therefore, $G$ has a fixed point. Finally, it is easy to prove that the conditions of Theorem 3 are satisfied, which implies that $G$ has a unique fixed point, i.e., there exists a unique solution of the Problem (20).

\section{COMPLIANCE WITH ETHICAL STANDARDS}

Conflict of interest: Authors declare that they have no conflict of interest.

\section{ACKNOWLEDGMENTS}

The first and third author would like to thank CSIR-HRDG Fund, under grant EMR-09/386(0059)/2017-EMR-1 and EMR-09/386(0064)/2019EMR-1 respectively, for financial support.

\section{REFERENCES}

[1] A. Alam, M. Imdad, Relation-theoretic contraction principle, Journal of Fixed Point Theory and Applications, 17 (4) (2015), 693-702.

[2] A. Alam, M. Imdad, Nonlinear contractions in metric spaces under locally T-transitive binary relations, Fixed Point Theory, 19 (1) (2018), 13-24.

[3] G. Prasad, R.C. Dimri, A. Bartwal, Fixed points of Suzuki contrative mappings in relational metric spaces, Rendiconti del Circolo Matematico di Palermo, 69 (3) (2019), $1347-1358$. 
[4] A. George, P. Veeramani, On some results in fuzzy metric space, Fuzzy Sets and Systems, 64 (1994), 395-399.

[5] M. Grabiec, Fixed points in fuzzy metric spaces, Fuzzy Sets and Systems, 27 (1988), 385-389.

[6] V. Gregori, J.J. Minana, D. Miravet, Contractive sequences in fuzzy metric spaces, Fuzzy Sets and Systems, 379 (2020), 125-133.

[7] V. Gregori, S. Morillas, A. Sapena, On a class of completable fuzzy metric spaces, Fuzzy Sets and Systems, 161 (2010), 2193-2205.

[8] V. Gregori, A. Sapena, On fixed-point theorems in fuzzy metric spaces, Fuzzy Sets and Systems, 125 (2002), 245-252.

[9] O. Hadžić, E. Pap, Fixed Point Theory in Probabilistic Metric Spaces, Springer, Dordrecht, 2001, 47-94.

[10] O. Hadžić, E. Pap, Fixed Point Theory in Probabilistic Metric Spaces, Kluwer Academic, Dordrecht, 2001.

[11] M. Jleli, E. Karapinar, B. Samet, On cyclic $(\psi, \phi)$-contraction in Kaleva-Seikkala's type fuzzy metric spaces, Journal of Intelligent \& Fuzzy Systems, 27 (2014), 2045-2053.

[12] M.S. Khan, M. Swaleh, S. Sessa, Fixed point theorems by altering distances between the point, Bulletin of the Australian Mathematical Society, 30 (1984), 1-9.

[13] B. Kolman, R.C. Busby, S. Ross, Discrete mathematical structures, 3rd ed. New Delhi: PHI Pvt. Ltd. 2000.

[14] I. Kramosil, J. Michalek, Fuzzy metric and statistical metric spaces, Kybernetika, 11 (5) (1975), 336-344.

[15] S. Lipschutz, Schaums outlines of theory and problems of set theory and related topics, New York: McGraw-Hill, 1964.

[16] J.J. Nieto, R. Rodríguez-López, Contractive mapping theorems in partially ordered sets and applications to ordinary differential equations, Order, 22 (2005), 223-239.

[17] G. Prasad, R.C. Dimri, Fixed point theorems for weakly contractive mappings in relational metric spaces with an application, The Journal of Analysis, 26 (1)(2018), 151-162.

[18] A.C.M. Ran, M.C.B. Reurings, A fixed point theorem in partially ordered sets and some applications to matrix equations, Proceedings of the AMS, 132 (5) (2004), 1435-1443.

[19] A.F. Roldán, López de Hierro, E. Karapinar, S. Manro, Some new fixed point theorems in fuzzy metric spaces, Journal of Intelligent \& Fuzzy Systems, 27 (5) (2014), $2257-2264$.

[20] A. Roldán, J. Martinez-Moreno, C. Roldán, On interrelationships between fuzzy metric structures, Iranian Journal of Fuzzy Systems, 10 (2) (2013), 133-150.

[21] B. Samet, M. Turinici, Fixed point theorems on a metric space endowed with an arbitrary binary relation and applications, Communications in Mathematical Analysis, 13 (2) (2012), 82-97.

[22] B. Schweizer, A. Sklar, Statistical metric spaces, Pacific Journal of Mathematics, 10 (1960), 314-334.

[23] Y. Shen, D. Qiu, W. Chen, Fixed point theorems in fuzzy metric spaces, Applied Mathematics Letters, 25 (2012), 138-141.

[24] S. Shukla, D. Gopal, W. Sintunavarat, A new class of fuzzy contractive mappings and fixed point theorems, Fuzzy Sets and Systems, 350 (2018), 85-94.

[25] P. Tirado, Contraction mappings in fuzzy quasi-metric spaces and [0,1]-fuzzy posets, Fixed Point Theory, 13 (1) (2012), 273-283. 
[26] M. Turinici, Contractive maps in locally transitive relational metric spaces, The Scientific World Journal, (2014), Article ID: 169358.

[27] M. Turinici, Contractive operators in relational metric spaces, In Handbook of Functional Equations, Springer Optimization and its Applications, ed. T. Rassias, New York, Springer, 95 (2014), 419-458.

[28] A. Bartwal, R.C. Dimri, G. Prasad, Some fixed point theorems in fuzzy bipolar metric spaces, Journal of Nonlinear Sciences and Applications, 13 (4) (2020), 196-204.

[29] A. Bartwal, R.C. Dimri, G. Prasad, On multidimentional fixed point theorems in ordered V-fuzzy metric spaces, International Journal of Scientific \& Technology Research, 8 (8) (2019), 1196-1203.

[30] G. Prasad, A. Tomar, R.C. Dimri, A. Bartwal, Coincidence theorems via contractive mappings in ordered non-Archimedean fuzzy metric spaces, Journal of the Korean Society of Mathematical Education Series B-pure and Applied Mathematics, 27 (4) (2020), 187-205.

[31] S. Shukla, D. Gopal, A.F. Roldán, Some fixed point theorems in 1-M-complete fuzzy metric-like spaces, International Journal of General Systems, 45 (7-8) (2016), 815-829.

[32] D. Gopal, C. Vetro, Some new fixed point theorems in fuzzy metric spaces, Iranian Journal of Fuzzy Systems, 11 (3) (2014), 95-107.

[33] D. Gopal, M. Abbas, C. Vetro, it Some new fixed point theorems in Menger PMspaces with application to Volterra type integral equation, Applied Mathematics and Computation, 232 (2014), 955-967.

[34] D. Gopal, Contributions to Fixed Point Theory of Fuzzy Contractive Mappings, Advances in Metric Fixed Point Theory, Springer Singapore, 2021.

[35] A. Bartwal, R.C. Dimri, A Common Fixed Point Theorem for a Pair of Mappings in Fuzzy Metric Spaces with An Application, Fixed Point Theory and Its Applications to Real World Problems, Nova Science Publishers, New York, 2021, 289-301.

\section{Ayush Bartwal}

Department of Mathematics

H.N.B. Garhwal University

UTTARAKHAND 246174

INDIA

E-mail address: ayushbartwal@gmail.com

\section{R.C. DimRi}

Department of Mathematics

H.N.B. Garhwal University

UTTARAKHAND 246174

INDIA

E-mail address: dimrirc@gmail.com

\section{ShIVAM RAWAT}

Department of Mathematics

H.N.B. Garhwal University

UTTARAKHAND 246174

INDIA

E-mail address: rawat.shivam09@gmail.com 